\title{
THE RATIO OF TOTAL TO SELECTIVE EXTINCTION IN THE DIRECTIONS OF SELECTED PLANETARY NEBULAE
}

\author{
JULIE H. LUTZ
}

Washington State University, Pullman, Washington, D.C., U.S.A.

\begin{abstract}
The ratio of total to selective extinction has been determined for the lines-of-sight to six planetary nebulae.
\end{abstract}

Values of the ratio of total to selective extinction $(R)$ were derived for the lines-ofsight to six planetary nebulae by using statistical distances to the nebulae (Cahn and Kaler, 1971) in conjunction with diagrams of color excess vs distance (hereafter called ce $-\mathrm{d}$ diagrams). The ce $-\mathrm{d}$ diagrams were constructed using early-type stars within 1.5 of each planetary (see Lutz and Lutz (1972) and Lutz (1973) for details of the observing program).

A value of $R$ was assumed for the line-of-sight to a given planetary. The color excess of the planetary was calculated from

$$
E(B-V)=2.03 \frac{c}{R},
$$

where $c$ is the extinction constant of the planetary and the constant 2.03 results from adopting the reddening law given by Whitford (1958). The extinction constant was derived by comparing the observed ratio of the radio and $\mathrm{H} \beta$ flux with a theoretically computed ratio. This requires an assumption of electron temperature. For this study the electron temperature was assumed to be $7000 \mathrm{~K}$. Raising the electron temperature from $7000 \mathrm{~K}$ to $10000 \mathrm{~K}$ would lower the value of $R$ ultimately derived by 0.2 . The radio fluxes were evaluated at $3 \mathrm{GHz}$, except in the cases of NGC $7026(8 \mathrm{GHz})$ and NGC $6741(10 \mathrm{GHz})$. The distance to each early-type program star was calculated using the assumed $R$, and a ce-d diagram was constructed. The distance to the planetary was found from the ce- $\mathrm{d}$ diagram. If the distance did not agree with the Cahn and Kaler distance, then another value of $R$ was tried. This method introduced an uncertainty of \pm 0.1 to \pm 0.2 into the value of $R$ derived.

TABLE I

Ratio of total to selective extinction

\begin{tabular}{lrr}
\hline Nebula & \multicolumn{1}{c}{$l$} & \multicolumn{1}{c}{$R$} \\
\hline NGC 6741 & $34^{\circ}$ & $>2.0$ \\
NGC 6894 & $70^{\circ}$ & 3.2 \\
NGC 7026 & $89^{\circ}$ & 3.0 \\
NGC 7354 & $108^{\circ}$ & 4.0 \\
IC 1747 & $130^{\circ}$ & $>3.1$ \\
IC 289 & $139^{\circ}$ & 4.6 \\
\hline
\end{tabular}


The value of $R$ for the line-of-sight to each planetary is shown in Table I. NGC 6741 and IC 1747 are optically thick, so the values of $R$ for these nebulae are lower limits to the true values of $R$. A $50 \%$ change in any of the Cahn and Kaler distances would produce a change of about 1.0 in the value of $R$ derived.

\section{References}

Cahn, J. H. and Kaler, J. B.: 1971, Astrophys. J. Suppl. 22, 319.

Lutz, J. H.: 1973, Astrophys. J. 181, 135.

Lutz, J. H. and Lutz, T. E.: 1972, Astron. J. 77, 376.

Whitford, A. E.: 1958, Astron. J. 63, 201. 